\title{
Health-aware Model Predictive Control including Fault-tolerant Capabilities for Drinking Water Transport Networks
}

\author{
Fatemeh Karimi Pour ${ }^{1}$ and Vicenç Puig ${ }^{1}$
}

\begin{abstract}
This paper presents a health-aware Model Predictive Control (MPC) including fault-tolerant capabilities for drinking water transport networks. When a fault has occurred, the predictive controller must be redesigned to deal with the fault. This is done by considering the system reliability that is incorporated into the MPC model. The inclusion of components and system reliability in the MPC model is done through the Linear Parameter Varying (LPV) modelling approach with the aim of maximizing the availability of the system by considering system reliability. As a result, the MPC design is modified by considering the reliability model such that additionally to achieve the best achievable performance after the fault, the controller try to preserve the remaining useful life. The solution to the optimization problem related to the MPC problem is achieved by solving a series of Quadratic Programming (QP) problems thanks to the proposed LPV formulation. The proposed approach is applied to a part of a real drinking water transport network of Barcelona for demonstrating the performance of the method.
\end{abstract}

\section{INTRODUCTION}

Drinking Water Networks (DWNs) are critical infrastructures in urban environments. These networks require advanced supervisory-control approaches to guarantee and maintain optimal performance even in faulty situations. Water consumption can change in both the short and the long term, ordinarily represented by different time-based models according the zone [1]. Therefore, better modelling and forecasting of demands will improve the modelling and control of DWNs. DWNs are multivariable dynamic constrained systems that are created by the interconnection of several subsystems (tanks, actuators, sources, intersection nodes and consumer sectors) [2]. Besides, its optimal management, that can be expressed as a multi-criteria problem, is an important challenge. Then, these complications of DWNs has led to the use of some variants of model predictive control (MPC) as a supervisory control approach [3]. Concerning the optimal control approaches for managing water systems, standard MPC is not implemented classically because there is no reference to be tracked. Regularly, standard MPC is expressed as an optimization problem that penalizes the tracking error. Alternatively, Economic MPC (EMPC) provides a systematic and suitable approach for managing systems by optimizing economic performance [4].

The MPC of DWNs needs to operate efficiently even in faulty situations. This problem calls for the use of a faulttolerant control (FTC) approach after a fault is diagnosed to avoid the MPC stopping every time a fault appears [5].

\footnotetext{
${ }^{1}$ F. Karimi Pour and V. Puig are with Institut de Robòtica i Informàtica Industrial (CSIC-UPC), C/. Llorens i Artigas 4-6, 08028 Barcelona, Spain vicenc.puig@upc.edu
}

FTC includes a variety of control approaches, which keep control systems in the appearance of faults running in a safety mode with satisfactory performance degradation. The object of FTC is to maintain a system fully effective by designing its control system such that system performance can be kept close to acceptable levels and stability requirements can be guaranteed, not only when the system is in nominal situations but also in case of the appearance of system component faults. Thus, FTC should, at the very last, guarantee an admissible degraded performance [6].

Recently, the problem of system reliability and actuator lifetime preservation has been gaining more interest from the researcher community. In [7], for reducing the maintenance cost, the actuator lifetime is perceived as a controlled parameter where is achieved by applying a linear-quadratic optimal controller. An MPC strategy based on distributing the loads with redundant actuators is presented in [8], while forcing constraints to ensure that the accumulated actuator degradation will not appear at the unsafe level at the end of the process. In [9], authors designed a health-aware MPC controller based on fatigue-based prognosis that aims at minimizing the damage of components. On the other hand, the incorporation of fault tolerance in MPC has already been regarded by several studies. In [10], authors provided a general overview of how to embed fault tolerance in MPC. The inclusion of fault-tolerance in MPC has principally been presented by considering practical strategies regarding the application domain. In [11], fault tolerant MPC has been applied to sewage networks by considering an hybrid systems framework. A method for incorporating fault tolerance in MPC for smart grids to guarantee the suitable value of energy in storage devices and reliable coverage of essential consumer demand provided [12]. In [2], an off-line approach to reliability based reconfiguration of an EMPC controller by structural analysis of the faulty actuators is proposed.

One disadvantage of the previous approaches to reliabilitybased MPC including fault-tolerance is that they consider the reliability at the actuator level but not at the system level, i.e. by taking into account the interconnection topology because of the non-linearity of the resulting constraints leading to the use of non-linear MPC (NMPC). NMPC is computationally expensive and, there is no guarantee that the solution of the optimization problem is the global one. Besides, the evaluation of the reliability was done off-line being necessary more time to recalculate the reliability model and modifying the controller by means structural analysis. Thus, by transforming the nonlinear problem to a quadratic problem by a linearization method is one way for solving the 
optimization problem in case of a nonlinear system. But, the system is modelled by an incremental model that has to be linearized again at each iteration. Alternatively, an approach has been recently developed using Linear Parameter Varying (LPV) models [13]. LPV models are a class of linear models whose state-space matrices depend on a set of time-varying parameters that can be measured/estimated on-line. The main benefit of LPV models is that the system nonlinearities are embedded in the varying parameters transforming the nonlinear system into a linear-like system with varying parameters [14].

The main contribution of this paper is to provide a healthaware MPC controller including fault-tolerance that uses on-line reliability evaluation for deciding the best control strategy after the fault occurrence. The MPC controller is redesigned to deal with the fault minimizing the degradation of performance and reliability. By using the LPV framework, the system reliability is incorporated into the MPC model. The augmented model that includes both the reliability and DWN models is expressed as an LPV model where at each time instant the scheduling parameters are updated. In this way, the MPC controller design can be modified to achieve the best achievable performance by updating the model reliability according to the control actions. Therefore, the control inputs are created to satisfy the control objectives and simultaneous to increase the lifespan and reliability of the system components. The proposed approach is tested in a part of the Barcelona water transportation network.

The remainder of the paper is organized as follows. The MPC controller for the transport DWN that includes fault tolerance capabilities is introduced in Section II. In Section III, the system reliability and reliable-aware MPCLPV including fault tolerance is provided. The results of an application of this approach based on the Barcelona case study are presented in Section IV. Finally, Section V concludes with some suggestions of research lines for future work.

\section{MPC OF DWN WITH FAULT-TOLERANT CAPABILITIES}

\section{A. Flow-based control-oriented model}

Several modeling methods dealing with DWNs have been proposed in the literature depending if the transportation or distribution layer is considered. (see, e.g., [15], [16]). In this paper, since the transportation layer is considered, a controloriented modeling approach that is based on a flow model is considered that follows the principles introduced by the authors in [16]. A DWN is a network which comprises a set of pressurized pipes, water tanks at different elevations, a number of pumping stations and valves to manage water flows, pressure, and elevation to supply water to consumers. Consider a general DWN as represented by a directed graph $G(\nu, \varepsilon)$, where a set of elements, i.e., $n_{d}$ sinks, $n_{s}$ sources, $n_{x}$ storage tanks and $n_{q}$ intersection nodes are represented by $v \in \nu$ vertices, which are connected by $a \in \varepsilon$ links. According to the network function, water is controlled in the network by $n_{u}$ flow actuators (i.e., valves and pipes), stored in tanks, from particular origin locations to specific target locations. Following flow/mass balance principles, a discretetime model based on linear differential algebraic equations (DAEs) for all time instant $k \in \mathbb{Z}_{\geq 0}$ can be formulated for a given DWN as follows:

$$
\begin{aligned}
x(k+1) & =A x(k)+B u(k)+B_{d} d_{m}(k), \\
0 & =E_{u} u(k)+E_{d} d_{m}(k),
\end{aligned}
$$

where the difference equations in (1a) present the dynamics of the storage tanks, and the algebraic equations in (1b) describe the static relations in the network (i.e., mass balance at junction nodes). $A \in \mathbb{R}^{n_{x} \times n_{x}}, B \in \mathbb{R}^{n_{x} \times n_{u}}, B_{d} \in$ $\mathbb{R}^{n_{x} \times n_{d}}, E_{u} \in \mathbb{R}^{n_{d} \times n_{u}}, E_{d} \in \mathbb{R}^{n_{d} \times n_{d}}$ and $C \in \mathbb{R}^{n_{y} \times n_{x}}$ are time-invariant matrices of that depends on the network topology. The system is subject to hard input and state as

$$
\begin{array}{ll}
x(k) \in \mathbb{X} \triangleq\left\{x(k) \in \mathbb{R}^{n_{x}} \mid \underline{x} \leq x(k) \leq \bar{x}\right\}, & \forall k \\
u(k) \in \mathbb{U} \triangleq\left\{u(k) \in \mathbb{R}^{n_{u}} \mid \underline{u} \leq u(k) \leq \bar{u}\right\}, & \forall k
\end{array}
$$

where vectors $\underline{x} \in \mathbb{R}^{n_{x}}$ and $\bar{x} \in \mathbb{R}^{n_{x}}$ determine the minimum and maximum possible state values of the system, respectively. Similarly $\underline{u} \in \mathbb{R}^{n_{u}}$ and $\bar{u} \in \mathbb{R}^{n_{u}}$ determine the minimum and maximum possible value of manipulated variables, respectively.

\section{B. EMPC Formulation of DWN}

Computing the input commands, ahead of time, to obtain the optimal performance of the network according to a set of control goals is the purpose of applying MPC techniques for managing water transportation networks. The control goal can formulated as the minimization of a convex multiobjective cost function including the following goals:

1) Economic costs Minimization: Minimizing the economic costs that include water production and electrical costs related to pumping is the main control objective of the DWN. Transporting drinking water to proper elevation levels by the network involves significant electricity costs due to pumping. Therefore, the cost function related to this objective can be expressed as

$$
\ell_{e}(k) \triangleq \alpha(k)^{\top} W_{e} u(k),
$$

where $\alpha(k) \triangleq\left(\alpha_{1}+\alpha_{2}(k)\right) \in \mathbb{R}^{n_{u}}, \alpha_{1} \in \mathbb{R}^{n_{u}}$ denotes a fixed water-production costs that are related to the water treatments and $\alpha_{2} \in \mathbb{R}^{n_{u}}$ corresponds to a time-varying water cost associated to pumping that varies in each time instant $k$ with respect to the dynamic electricity tariff. $W_{e}$ indicates the weighting term.

2) Safety Management: Regarding preserving water stock despite the change of water demands among two consecutive MPC iterations, an appropriate safety capacity for each storage tank is required to be maintained. A possible mathematical formulation for this goal can be formulated as follows

$$
\ell_{s}(k) \triangleq\left\{\begin{array}{lr}
\left\|x(k)-x_{s}\right\|_{2}, & \text { if } x(k) \leq x_{s} \\
0, & \text { otherwise }
\end{array}\right.
$$

where $x_{s}$ indicates the vector of the safety levels for all the tanks. The safety cost function can be expressed through 
a soft constraint by adding a slack variable $\xi$ that can be formulated as

$$
\ell_{s}(k) \triangleq \xi^{\top}(k) W_{s} \xi(k),
$$

where the next soft constraint is included $x(k) \geq x_{s}-\xi(k)$, and $W_{s}$ is diagonal positive definite matrix.

3) Smoothness of Control Actions: Pumps and valves are the considered actuators in a DWN. To guarantee the smoothing effect, the slew rate of the control actions among two sequential time instants is penalized according to

$$
\ell_{\Delta u}(k) \triangleq \Delta u(k)^{\top} W_{\Delta u} \Delta u(k)
$$

where $\Delta u(k) \triangleq u(k)-u(k-1)$, and $W_{\Delta u}$ is a diagonal positive definite matrix.

According to the network model (1), the MPC controller design is based on minimizing the following cost function in the prediction horizon $N_{p}$

$$
J=\sum_{l=0}^{N_{p}}\left(\ell_{e}(l \mid k)+\ell_{s}(l \mid k)+\ell_{\Delta u}(l \mid k)\right) .
$$

Following [2], the control problem is solved by obtaining the control law from a provided set of control laws $\mathcal{U}$, such that the controlled system reaches the control objectives $\mathcal{O}$ while its behaviour convinces a set of constraints $\mathcal{C}$.

\section{Inclusion the fault-tolerant abilities}

When faults are considered, the control problem $\langle\mathcal{O}, \mathcal{C}, \mathcal{U}\rangle$ described earlier will be reformulated to take into account their effect. By considering an active FTC strategy, two main strategies for adapting the MPC law to include fault tolerance can be applied: system reconfiguration and fault accommodation. In this paper, system reconfiguration approach is considered.

The system reconfiguration involves obtaining a new set of constraints $\mathcal{C}_{f}\left(\Theta_{f}\right)$ i.e. model, where $\Theta_{f}$ is the set parameters modified by the faults, such that the control problem $\left\langle\mathcal{O}, \mathcal{C}_{f}\left(\Theta_{f}\right), \mathcal{U}_{f}\right\rangle$ should be solved. This approach can be applied in case the fault detection and isolation (FDI) does not provide the fault estimation. Therefore, the faulty components are unplugged by the monitoring system and the control objectives must be achieved by using the non-faulty components. In the case of faulty actuators, the model (1) that used by the MPC controller is modified as follows:

$$
\begin{aligned}
x(k+1) & =A x(k)+\sum_{i \in I_{N}} B_{i} u_{i}(k)+B_{d} d(k), \\
0 & =\sum_{i \in I_{N}} E_{u, i} u_{i}(k)+E_{d} d(k),
\end{aligned}
$$

where $I_{N}$ is the subset of still-healthy actuators.

Note that by adapting the model (1) of the MPC controller using the system reconfiguration approach, the controller will consider the effect of the fault in the system model when calculating the control action $u_{0}^{*} \mid k$. Following [17], the differences of MPC with other control laws (e.g., pole placement and LQR ) is that the control should not be computed off-line for the given set of faults by creating a bank of controllers that must be gain-scheduled on-line taking to account the fault features. But, instead the reconfiguration of the MPC control law is done on-line by including the fault effect in the control model (8). However, the MPC controller might not be able to determine a control input neither the calculated control input can not steer the system to a satisfactory performance. Because of this, when applying an MPC controller the effect of the fault and the admissibility of the computed control input requires to be assessed on-line as discussed in [2].

\section{FAULT-TOLERANT HEALTH-AWARE MPC-LPV}

\section{A. Actuator fault-tolerance evaluation}

As discussed in [2], in case that a fault occurs, it may occur that

- the system has lost some of the components needed to continue with the control, or

- system performance is degraded to an unacceptable level and it is not worth to continue with system control by using fault-tolerant procedures.

When a reconfiguration strategy is applied, the faulty actuator is eliminated and the connectivity between sources and demands might be lost. This will affect both reliability and controllability. However, these structural properties do not consider the physical constraints of the system actuators. Therefore, in spite of that connectivity is maintained, the optimization problem related to the MPC may steer to the unfeasible solution, due to the reduction of capacity of the remaining actuators or the inadequate performance of the control loop. Therefore, to prevent from such problems, the actuator redundancy should be guaranteed by design. The redundancy assessment of actuator can done by using the reliability analysis. Actually, after a fault has occurred, the reliability model should be evaluated again. Hence, it necessary to redo the analysis of the reliability and it could be the fault affects a non-redundant actuator but also a critical one. In this case, if the fault affects a critical actuator, MPC can not to continue with the control of the system. On the other hand, if the fault affects the redundant actuator then it is still possible to continue the control.

\section{B. Reliability analysis algorithm}

Failure rate and reliability Concept: Let us first define the concept of failure rate which is important to obtain reliability. The failure rate, denoted by $\lambda$, represents the fraction of the density of the stochastic lifetime to the remainder function (i.e., conditional probability). In this paper, failure rates are determined from actuators under various levels of load respect to the applied control input. The most widely used relationship is based on assuming that actuator fault rates varied through the load by the following exponential law

$$
\lambda_{i}=\lambda_{i}^{0} \exp \left(\beta_{i} u_{i}(k)\right), \quad i=1,2, \ldots, m
$$


where $\lambda_{i}^{0}$ represents the baseline failure rate (nominal failure rate) and $u_{i}(k)$ is the control action a time $k$ for the $i$ th actuator. $\beta_{i}$ is a constant parameter that depends on the actuator characteristics.

From the mathematical point of view, reliability $R(t)$ is the probability that a system will be successful in the time interval from 0 to $t$. Besides, the unreliability $F(t)$ is determined as the probability that the component or system encounters the first failure or has failed one or more times among the time interval 0 to time $t$. Considering that the system (or component) is always in one of the two mentioned states, the following relationship is provided

$$
F(t)+R(t)=1 .
$$

In the useful life period, the component reliability at a certain time $t$ is given by

$$
R_{i}(t)=\exp \left(-\lambda_{i}^{0} t\right), \quad i=1,2, \ldots, m
$$

Hence, the evolution of the component reliability of a $i$ th system component in discrete-time can be computed as follows

$$
R_{i}(k+1)=R_{i}(k) \exp \left(-T_{s} \sum_{s=0}^{k+1} \lambda_{i}(s)\right), \quad i=1,2, \ldots, m
$$

where $\lambda_{i}(s)$ is the failure rate that is acquired from the $i$ th component under varying levels of load $u_{i}$ and $T_{s}$ is the sampling time.

System reliability modeling by using Markov's Processes: Let $e_{i}$ be a discrete random variable in the Markov process representing the state of the $i$-th component with two possible mutually exclusive states, i.e. up (Up) and down (Dn). The probabilistic state transition between the states is defined by:

$$
P_{M C}\left(e_{i}(k+1) \mid e_{i}(k)\right)=\left[\begin{array}{cc}
1-p_{12} & p_{12} \\
0 & 1
\end{array}\right]
$$

where $p_{12} \cong \lambda \Delta t$ and $\Delta t$ represents the time interval, and $p_{12}$ can be interpreted as the probability that the component goes from state Up to Dn after $\Delta t$.

Therefore, it is clear that the failure rate is the probability of component state to be Dn at instant time $k+1$ given that its state was Up at time $k$, that is:

$$
\lambda_{i}(k)=\operatorname{Pr}\left(e_{i}(k+1)=D n \mid e_{i}(k)=U p\right),
$$

and allows to compute the component reliability using:

$$
R_{i}(k+1)=\operatorname{Pr}\left(e_{i}(k+1)=U p\right) .
$$

Therefore, the overall system reliability $R_{G}(k)$ depends on the actuators configuration which can be computed as

$$
R_{g}(k)=1-\prod_{j=1}^{s}\left(1-\prod_{i \in P_{s, j}} R_{i}(k)\right)
$$

where $j=1, \ldots, s$ is the number of minimal paths $P_{s}$. A minimal path is a set of components such that the elimination of one of them will lead the set not to be a successful path.
Notice that the evaluation of this probability could be quite involved using standard reliability analysis but thanks to the Markov chain mechanism is quite simple.

In order to integrate the reliability in the MPC model as an additional state variable, a transformation is required that allows to compute reliability in a linear-like form. The proposed transformation is based on using the logarithm of (17). As stated in (11), (17) can be rewritten as

$$
\log \left(Q_{G}(k)\right)=\log \left(\prod_{j=1}^{s}\left(1-\prod_{i \in P_{s, j}} R_{i}(k)\right)\right)
$$

and by introducing a change of variable

$$
z_{j}(k)=1-\prod_{i \in P_{s, j}} R_{i}(k),
$$

\section{Inclusion of Fault-tolerant Capabilities}

In this section, the integration of the reliability model in the MPC controller augmenting the DWN model is proposed. As previously discussed, when a fault occurs, the MPC law is modified to cope with the fault, as discussed in Section 2.3. As explained in [18], the value of the actuator failure rate changes because the control action should be increased in order to compensate for the fault effect. In this case, energy consumption increases and the value of the failure rate also increases due to the actuator load increment. Thus, there is an interplay between maintaining closed-loop performance and reliability. To maintain the desired performance, the relationship between the actuator load increment and reliability can be established. Therefore, a new objective can be included in the MPC controller that aims to preserve the system reliability by considering the reliability model (17).

By following this procedure, the augmented MPC model including fault can be formulated as follows

$$
\begin{aligned}
x_{g}(k+1) & =A_{g} x_{r}(k)+\sum_{i \in I_{N}} B_{g, i} u_{i}(k)+B_{d, g} d_{m}(k), \\
0 & =\sum_{i \in I_{N}} E_{u, i} u_{i}(k)+E_{d} d_{m}(k),
\end{aligned}
$$

where the state and output vector are given by $x_{g}=$ $\left[x, \log \left(Q_{g}\right), \log \left(R_{1}\right), \ldots, \log \left(R_{i}\right)\right]^{T}$ and $y_{g}=\left[y, \log \left(Q_{g}\right)\right]^{T}$, respectively. The augmented matrices are defined as

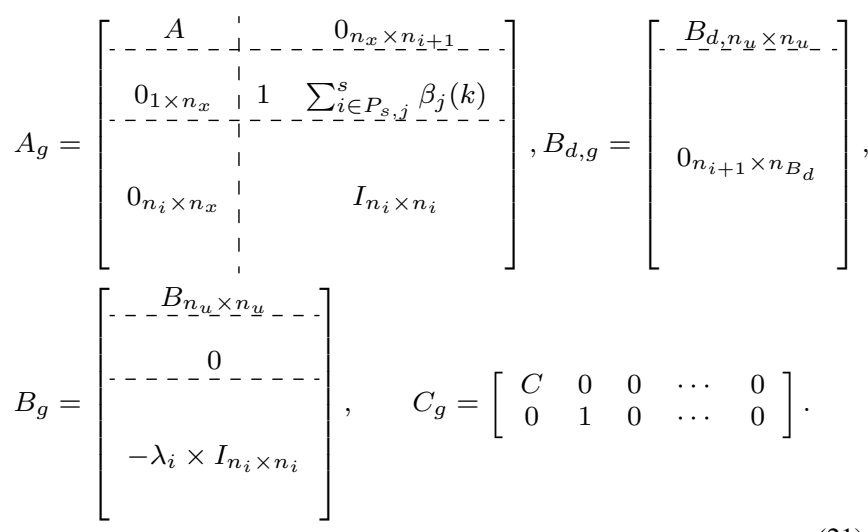


where $\ell_{R g}(k) \triangleq Q_{g}^{\top} w_{3} Q_{g}$ is additional objective with the corresponding weight $w_{3}$ into the MPC cost function to maximize the system reliability. Considering the control action $u_{i}(k)$ as the scheduling variable related to each actuator and state in the augmented MPC model, it can be considered (20) as an LPV model. In this way, by following the MPCLPV approach in [1], the MPC optimization problem can be formulated as a QP problem by using an estimation of scheduling variables.

\section{APPLICATION EXAMPLE}

The system used as a case study is a part selected from the Barcelona DWN that reported in [15] (see Figure 1). The reliable-aware MPC-LPV including the effect of fault proposed in the previous section has been implemented in simulation.

The objective of the MPC, as has been explained before, is to minimize the multi-objective cost function (7) with and without the reliability objective. The prediction horizon is 24 hours because the system and also the electrical tariff have periodicity of 1 day. The analysis is exhibited for a time period of 12 day ( 288 hours) with sampling time of 1 hour. The weights of the cost function are $W_{e}=$ $100, W_{s}=1, W_{\Delta_{u}}=1$ and $W_{R_{g}}=10$. The tuning of these parameters is adjusted according to that the objective with the highest preference is the economic cost, which must be minimized maintaining proper levels of safety volumes and control action smoothness and the same time should cover the effect of the fault and preserve the system reliability. The simulation results based on real data are obtained using the CPLEX optimization package and Matlab R2015b (64 bits), running in a PC Intel(R) Core(TM)i7-5500 CPU at $2.4 \mathrm{GHz}$ with 12 GB of RAM.

The FTC reconfiguration strategy of the MPC controller based on proposed approach is now illustrated for the case of a fault in actuator 29, which is redundant. Fig. 2 presents the volume behaviour of tank 9, which is supplied by two actuators: 33 and 29. According to Fig.2, it can be observed that in a non-faulty situation, the volume of tank 9 shows

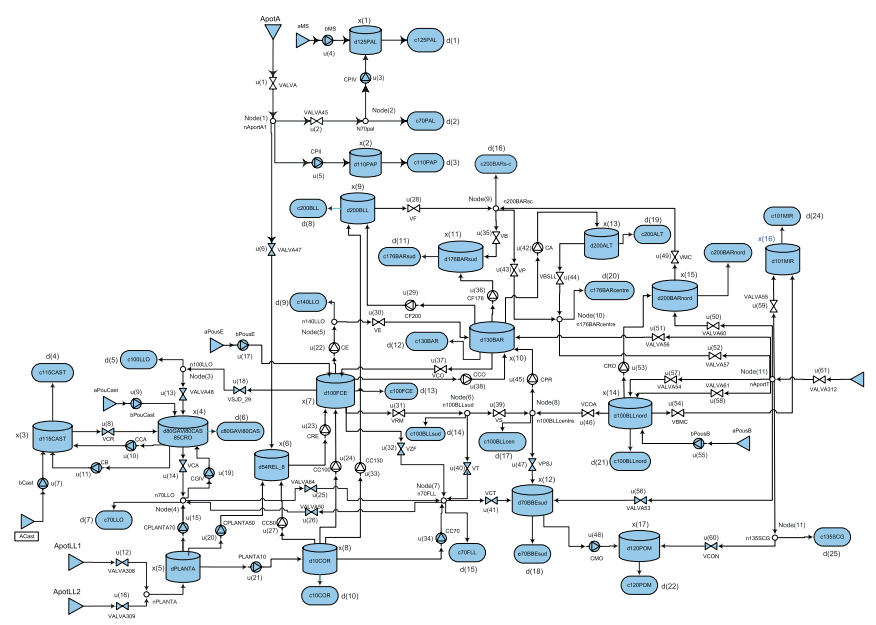

Fig. 1: Barcelona drinking water network a repeated pattern (filling when pumping is cheaper and emptying otherwise) to provide the water demand. But, when a fault occurs (at $T=150 \mathrm{~h}$ ), if the MPC controller is not reconfigured, tank 33 volume drops to zero at $T=157 \mathrm{~h}$ and demand is not satisfied anymore (unfeasible solution). But, if the MPC controller is reconfigured by removing the faulty actuator 29 from the control model and in the reliability model, the tank level can supply the demand. However, the tank volume reduces with time, meaning that the faulty actuator should be fixed. Figures 3 and 4 show the behaviour of actuators 29 and 33. Actuator 33 starts to deliver more flow to compensate for the faulty actuator 29 that is removed.

Furthermore, the analysis of total system reliability with and without the reliability objective in the controller are presented in Fig. 5. It can be seen that by considering the reliability objective the life time of the network is improved from 0.7994 to 0.933 and it is about $17.02 \%$ of reliability improvement in the MPC-LPV controller with the reliability objective.

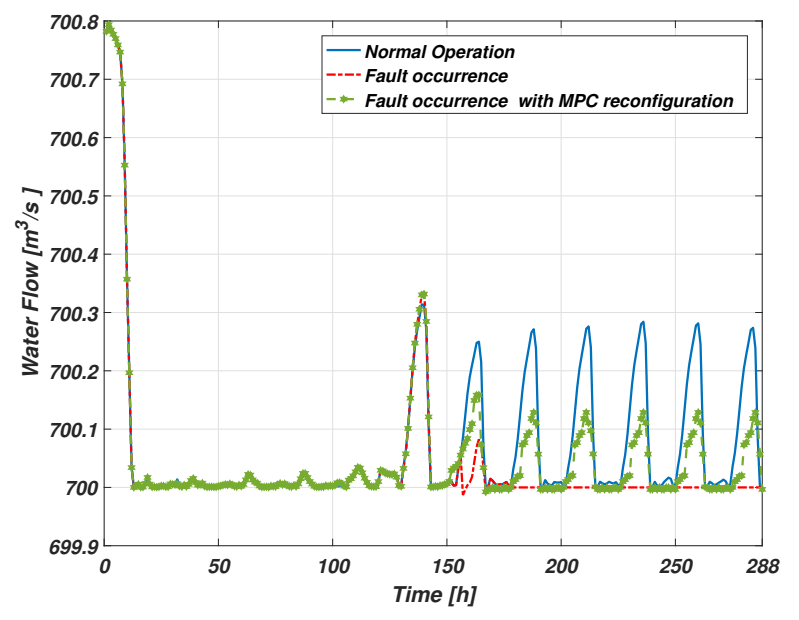

Fig. 2: Evolution of tank 9 with MPC using reconfiguration.

\section{CONCLUSION}

In this paper, the inclusion the fault-tolerant capabilities in a health-aware MPC controller for drinking the water network is proposed. The proposed approach allows a degradation analysis of the system to be implemented in terms of performance and reliability. By using the LPV framework, the system reliability is incorporated into the control algorithm. In this way, the MPC controller is modified to achieve the best achievable performance by updating the model reliability according to the control action. Moreover, the proposed health-aware MPC-LPV approach is efficiently solved iteratively by a series of QP problems that uses an update MPC model updated via the scheduling parameters calculated at each time instant. The results obtained show that the total system reliability of the DWN network is maximized with the proposed controller including the reliability objective. Finally, the proposed approach has been successfully tested in the Barcelona water network. Future research will 


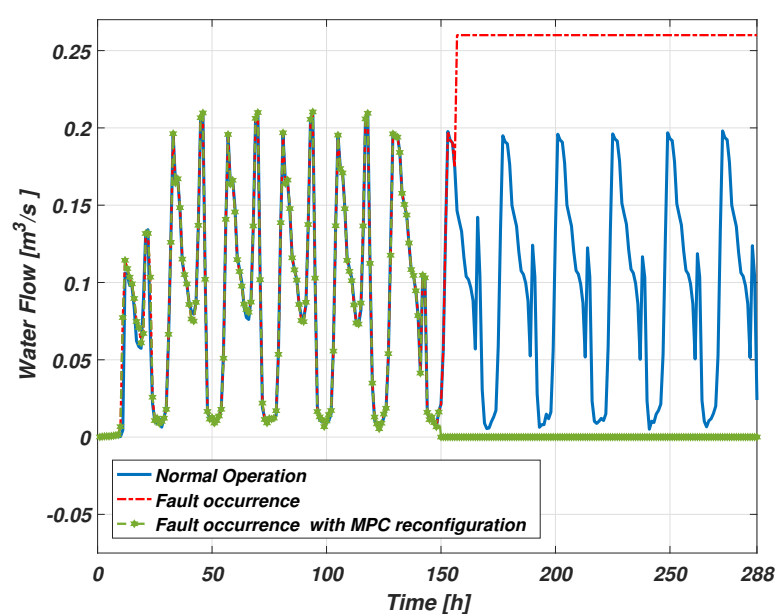

Fig. 3: Water flow in actuator 29 with MPC using reconfiguration.

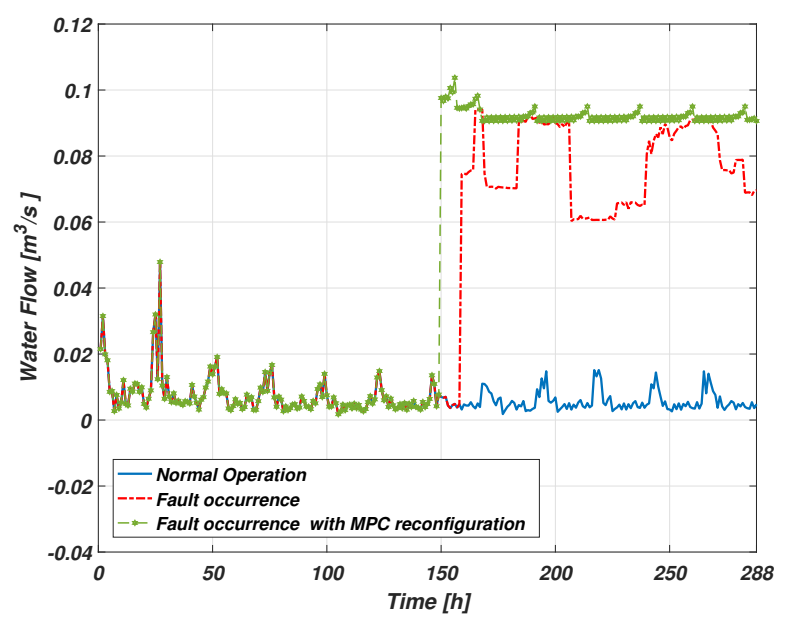

Fig. 4: Water flow in actuator 33 with MPC using reconfiguration.

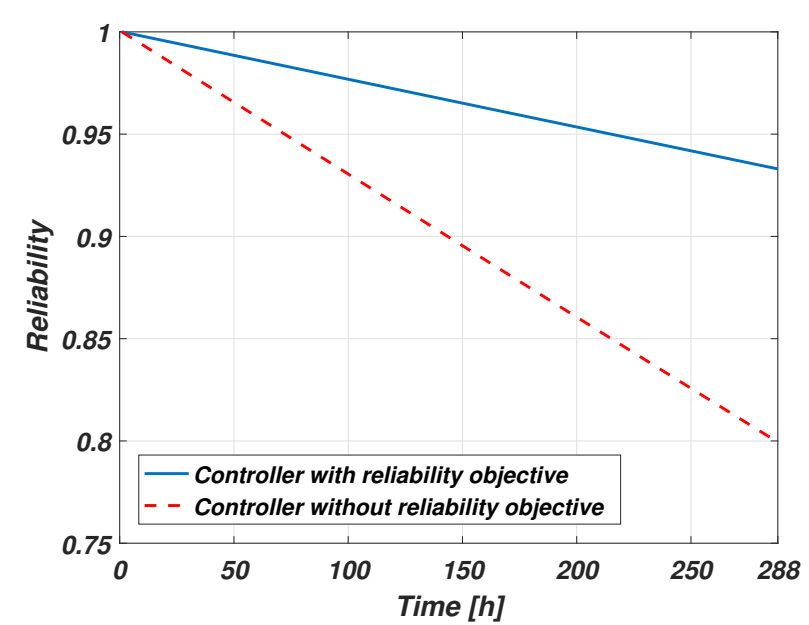

Fig. 5: Evaluation of total system reliability . focus on the study of FTC accommodation strategy of the MPC controller.

\section{ACKNOWLEDGMENT}

This work has been partially funded by the Spanish State Research Agency (AEI) and the European Regional Development Fund (ERFD) through the project SCAV (ref. MINECO DPI2017-88403-R).

\section{REFERENCES}

[1] F. Karimi Pour, V. Puig, and G. Cembrano, "Economic health-aware lpv-mpc based on system reliability assessment for water transport network," Energies, vol. 12, no. 15, p. 3015, 2019.

[2] D. Robles, V. Puig, C. Ocampo-Martinez, and L. E. Garza-Castañón, "Reliable fault-tolerant model predictive control of drinking water transport networks," Control Engineering Practice, vol. 55, pp. 197211, 2016.

[3] F. Karimi Pour, V. Puig, and G. Cembrano, "Health-aware lpv-mpc based on system reliability assessment for drinking water networks," in 2018 IEEE Conference on Control Technology and Applications (CCTA). IEEE, 2018, pp. 187-192.

[4] M. Ellis, H. Durand, and P. D. Christofides, "A tutorial review of economic model predictive control methods," Journal of Process Control, vol. 24, no. 8, pp. 1156-1178, 2014.

[5] V. Puig, "Admissible model matching fault tolerant control based on lpv fault representation," Journal of Instrumentation, Automation and Systems, vol. 2, no. 3, pp. 81-90, 2016.

[6] M. Blanke, M. Kinnaert, J. Lunze, and M. Staroswiecki, Diagnosis and Fault-Tolerant Control, 2nd ed. Berlin, Heidelberg: Springer-Verlag, 2006.

[7] L. Gokdere, S. L. Chiu, K. J. Keller, and J. Vian, "Lifetime control of electromechanical actuators," in Aerospace Conference, 2005 IEEE. IEEE, 2005, pp. 3523-3531.

[8] E. B. Pereira, R. K. H. Galvão, and T. Yoneyama, "Model predictive control using prognosis and health monitoring of actuators," in Industrial Electronics (ISIE), 2010 IEEE International Symposium on. IEEE, 2010, pp. 237-243.

[9] F. Karimi Pour, V. Puig, and C. Ocampo-Martinez, "Multi-layer healthaware economic predictive control of a pasteurization pilot plant," International Journal of Applied Mathematics and Computer Science, vol. 28, no. 1, pp. 97-110, 2018.

[10] E. F. Camacho, T. Alamo, and D. M. de la Peña, "Fault-tolerant model predictive control," in 2010 IEEE 15th Conference on Emerging Technologies \& Factory Automation (ETFA 2010). IEEE, 2010, pp. $1-8$.

[11] C. Ocampo-Martinez and V. Puig, "Fault-tolerant model predictive control within the hybrid systems framework: Application to sewer networks," international journal of adaptive control and signal processing, vol. 23, no. 8, pp. 757-787, 2009.

[12] M. Morato, P. Mendes, J. Normey-Rico, and C. Bordons, "Lpv-mpc fault-tolerant energy management strategy for renewable microgrids," International Journal of Electrical Power Energy Systems, vol. 117, p. 105644, 2020.

[13] P. Bumroongsri and S. Kheawhom, "Mpc for lpv systems based on parameter-dependent lyapunov function with perturbation on control input strategy," Engineering Journal, vol. 16, no. 2, pp. 61-72, 2012.

[14] F. Karimi Pour, V. Puig Cayuela, and C. Ocampo-Martínez, "Comparative assessment of lpv-based predictive control strategies for a pasteurization plant," in 4th-2017 International Conference on Control, Decision and Information Technologies, 2017, pp. 1-6.

[15] C. Ocampo-Martínez, V. Puig, G. Cembrano, R. Creus, and M. Minoves, "Improving water management efficiency by using optimization-based control strategies: the barcelona case study," Water science and technology: water supply, vol. 9, no. 5, pp. 565-575, 2009.

[16] G. Cembrano, J. Quevedo, M. Salamero, V. Puig, J. Figueras, and J. Mart1, "Optimal control of urban drainage systems. a case study," Control engineering practice, vol. 12, no. 1, pp. 1-9, 2004.

[17] J. M. Maciejowski, Predictive control: with constraints. Pearson education, 2002.

[18] F. Guenab, P. Weber, D. Theilliol, and Y. Zhang, "Design of a fault tolerant control system incorporating reliability analysis and dynamic behaviour constraints," International Journal of Systems Science, vol. 42, no. 1, pp. 219-233, 2011. 